\title{
Estudo do rompimento de fios sob a ação de impactos de tração
}

\author{
A study of wire rupture under traction impacts \\ B.N. de Albuquerque ${ }^{1}$, A.G. Lopes Jr. ${ }^{1}$, F.C. Santos*1,2 \\ ${ }^{1}$ Instituto de Ciência e Tecnologia, Universidade Federal dos Vales do Jequitinhonha e Mucuri, \\ Diamantina, MG, Brasil \\ ${ }^{2}$ Instituto de Física, Universidade Federal do Rio de Janeiro, Rio de Janeiro, RJ, Brasil
}

Recebido em 14 de janeiro de 2016. Revisado em 9 de março de 2016. Aceito em 19 de março de 2016

Neste trabalho estudamos o rompimento de fios metálicos submetidos à ação de impactos de tração. Obtivemos dados experimentais para relacionar a massa $M$ e a velocidade $V$ de um corpo que causa um impacto de tração em um fio na condição crítica de rompimento. Usando o teorema $\Pi$ de Buckingham da análise dimensional, determinamos uma expressão para a função $M=F(V)$ e comparamos com os dados experimentais. Também fizemos uma análise teórica com o modelo mais simples possível e verificamos que ele é compatível com os dados experimentais.

Palavras-chave: Análise Dimensional, Elasticidade, Teorema Trabalho-Energia.

In the present work we analyze the rupture of metallic wires under the action of stressing impacts. We have collected experimental data in order to relate the mass $M$ and speed $V$ of the body causing the stressing impact in a wire on the threshold of rupture. By making use of П-Buckingham's theorem of dimensional analysis we have determined an expression for the function $M=F(V)$ and have compared it to simplest possible model of the phenomenon and verified that it is compatible with experimental data.

Keywords: Dimensional analysis, elasticity, work-energy theorem.

\section{Introdução}

O estudo de deformações em materiais é largamente apresentado em diversos livros especializados e em artigos técnicos publicados nas revistas de Física dos materiais e de Engenharia. A enorme capacidade da matéria se organizar nas mais variadas combinações e formas se reflete na teoria, exigindo esforços coordenados nas áreas experimental e teórica. A elasticidade, a plasticidade, a fluidez, a viscidez, etc., não são características isoladas da matéria, mas sim propriedades que aparecem simultaneamente nos fenômenos reais. Neste trabalho, vamos nos restringir às tensões de deformação, especificamente ao limite de deformação de fios metálicos sob a ação de impactos de tração que causam seu rompimento. O principal objetivo desse trabalho é aplicar, de forma didática, a análise dimensional nesse problema rela- tivamente simples, frisando sua importância como uma ferramenta auxiliar bastante eficaz na solução de problemas teóricos e práticos. Também por motivos didáticos, os dados experimentais usados para testar os resultados teóricos referem-se somente a um fio fino de cobre de comprimento fixo.

Na seção 2, definiremos o problema a ser estudado, discutiremos brevemente o teorema fundamental da análise dimensional no contexto do nosso problema e obteremos as relações entre as grandezas relevantes. Na seção 3, descreveremos o equipamento usado e como foi feito o controle das variáveis medidas. $\mathrm{Na}$ seção 4, veremos como um modelo teórico bem simples para a relação entre a tensão e a deformação com somente um parâmetro pode se ajustar aos dados experimentais obtidos.

$\mathrm{Na}$ última seção, analisaremos os dados experimentais que obtivemos e mostraremos que o modelo

*Endereço de correspondência: filadelf@if.ufrj.br. 
teórico proposto na seção 4 é bastante razoável para fios metálicos nas condições estudadas.

\section{A análise dimensional do problema}

Inicialmente, com o objetivo de facilitar a discussão teórica, descreveremos o sistema estudado de forma simplificada sem qualquer preocupação com as condições reais de realização experimental e, em seguida, demonstraremos o teorema $\Pi$ de Buckingham no contexto do nosso problema. O sistema proposto, nesse trabalho, consiste de um fio metálico fino suspenso em um ponto fixo $A$ de uma superfície horizontal rígida e imóvel. Na extremidade inferior $B$ do fio fixamos uma pequena trava, ilustrada na figura 1. para permitir o impacto com uma massa $M$ cuja velocidade no instante do impacto é $V$.

Após o impacto, a massa $M$, devido à sua própria inércia e ao seu peso, começa a deformar o fio metálico. Para evitar a ação do peso, poderíamos formular o problema com o fio na horizontal e a massa $M$ se movendo sobre um plano horizontal. Entretanto, essa simplificação teórica não seria vantajosa do ponto de vista experimental, pois a presença de atritos dificultaria enormemente a realização da experiência.

Consideramos um fio metálico cuja tensão de ruptura é $T_{R}$ e deformação de ruptura é $d_{R}$ e queremos determinar o valor mínimo da massa de um bloco capaz de romper o fio quando sua velocidade no momento do impacto é $V$. Podemos supor que essa massa depende da tensão de ruptura, da deformação de ruptura, da velocidade no instante do impacto e da aceleração da gravidade $g$, ou seja,

$$
M=F\left(T_{R}, d_{R}, V, g\right)
$$

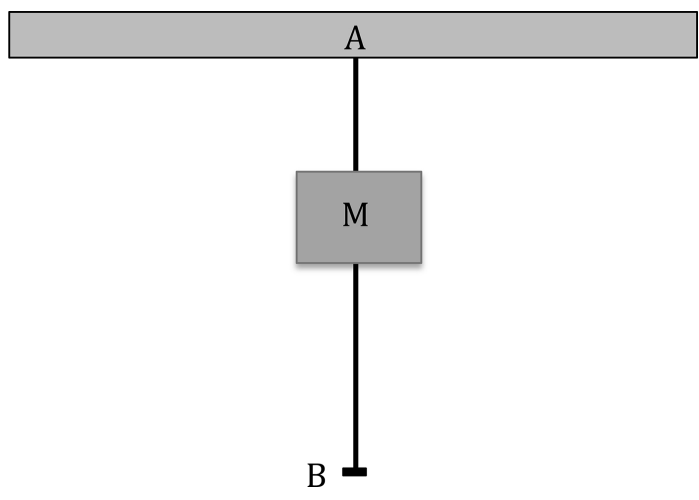

Figura 1: Modelo simplificado da experiência
As variáveis independentes em (1) não são dimensionalmente independentes porque estamos considerando que o número máximo de dimensões independentes no âmbito da mecânica é três. Então, podemos determinar as combinações dessas variáveis que são adimensionais. Denotando as dimensões das grandezas primárias massa, comprimento e tempo respectivamente por $M, L$ e $T$, podemos determinar as dimensões das variáveis independentes em (1):

$$
\begin{aligned}
{\left[T_{R}\right] } & =M L T^{-2} \\
{\left[d_{R}\right] } & =L \\
{[V] } & =L T^{-1} \\
{[g] } & =L T^{-2} .
\end{aligned}
$$

As combinações adimensionais que podem ser construídas com as variáveis independentes são determinadas pela seguinte condição:

$$
\left[T_{R}\right]^{\alpha}[V]^{\beta}\left[d_{R}\right]^{\gamma}[g]^{\delta}=1
$$

ou, usando as equações (2), podemos escrever a equação (3) na forma

$$
[M]^{\alpha}[L]^{\alpha+\beta+\gamma+\delta}[T]^{-2 \alpha-\beta-2 \delta}=1 .
$$

Tendo em vista que as dimensões $L, M$ e $T$ são independentes, devemos anular os expoentes para termos uma grandeza adimensional. Assim, obtemos as seguintes equações:

$$
\begin{array}{r}
\alpha=0, \\
\alpha+\beta+\gamma+\delta=0, \\
-2 \alpha-\beta-2 \delta=0 .
\end{array}
$$

A solução geral desse sistema de equações é

$$
\begin{array}{r}
\alpha=0, \\
\beta=-2 \delta \\
\gamma=\delta
\end{array}
$$

Assim as combinações adimensionais que podem ser construídas são da forma $\left(\frac{d_{R} g}{V^{2}}\right)^{\delta}$. Contudo, sem perda de generalidade, podemos escolher $\delta=1 \mathrm{e}$ definir a variável adimensional

$$
\Pi_{1}=\frac{g d_{R}}{V^{2}} .
$$

Também podemos determinar a combinação das variáveis independentes em (1) que tem a mesma 
dimensão da variável dependente. Nesse caso, considerando que o segundo membro da equação (3) seja $M$, obtemos as seguintes equações:

$$
\begin{array}{r}
\alpha=1 \\
\alpha+\beta+\gamma+\delta=0 \\
-2 \alpha-\beta-2 \delta=0 .
\end{array}
$$

Resolvendo o sistema acima, para $\delta=0$, obtemos $\alpha=1, \beta=-2, \gamma=1$ e definimos a variável adimensional

$$
\Pi_{0}=\frac{M}{\frac{T_{R} d_{R}}{V^{2}}} .
$$

Considerando a variável $\Pi_{0}$ como independente e substituindo a variável independente $g$ pela variável $\Pi_{1}$, podemos escrever (1) na forma

$$
\Pi_{0}=F\left(T_{R}, d_{R}, V, \Pi_{1}\right),
$$

onde $F$ representa uma nova função diferente daquela que aparece em (1). Notando que uma mudança do sistema de unidades não altera os valores das variáveis adimensionais, mas altera os valores das variáveis dimensionais, podemos afirmar que a variável adimensional $\Pi_{0}$ só pode depender da variável adimensional $\Pi_{1}$, ou seja,

$$
\Pi_{0}=F\left(\Pi_{1}\right) .
$$

A equação (11) é a expressão final do teorema $\Pi$ de Buckingham. A demonstração geral do teorema pode ser encontrada em [1] e [2]. Para um texto completo e com muitas aplicações em diversas áreas, pode-se consultar 3 e, finalmente, pode-se consultar o excelente texto [4] em português. Voltando para a equação (11), podemos, tendo em vista as definições das variáveis adimensionais (9) e (7), reescrevê-la na forma

$$
M=\frac{T_{R} d_{R}}{V^{2}} F\left(\frac{g d_{R}}{V^{2}}\right) .
$$

A grande vantagem da análise dimensional está no fato de que a função $F$ em (12) pode ser determinada, experimentalmente, variando somente uma única variável independente do conjunto de variáveis independentes que definem $\Pi_{1}$.

\section{Montagem experimental}

Nessa seção, vamos descrever a montagem experimental que utilizamos e analisar os dados obtidos.
Usando barras de madeira, construímos uma estrutura rígida na forma de um paralepípedo de aproximadamente 2, $0 \mathrm{~m}$ de altura com bases quadradas de $50 \mathrm{~cm}$ de lado. Na parte superior dessa estrutura, fixamos um suporte de madeira (mais uma barra de madeira) ao longo da diagonal do quadrado. Prendemos uma argola parafuso no meio desse suporte para suspender verticalmente os fios metálicos. A Figura (2) ilustra a montagem da estrutura.

Agora vamos descrever o procedimento experimental. O fio de cobre usado na experiência foi retirado de um cabo elétrico flexível, vendido em lojas comerciais de material elétrico, composto por 27 fios finos de 0,30(3) $\mathrm{mm}$ de diâmetro, o algarismo entre parentêsis representa a incerteza sobre o último algarismo de valor na medida: $0,30(3) \mathrm{mm}=$ $(0,30 \pm 0,03) \mathrm{mm}$. Cortamos um pedaço desse fio suficientemente comprido para deixar $80 \mathrm{~cm}$ de comprimento suspenso após enrolar firmemente uma de sua extremidades na argola parafuso. É importante trabalhar com um comprimento fixo do fio para garantir que a deformação de ruptura seja a mesma sempre que trocarmos o fio. Para medirmos a velocidade da massa $M$ no instante do impacto, optamos por prender a massa $M$ à extremidade do fio e deixála cair de uma altura $H$ acima do ponto inferior do

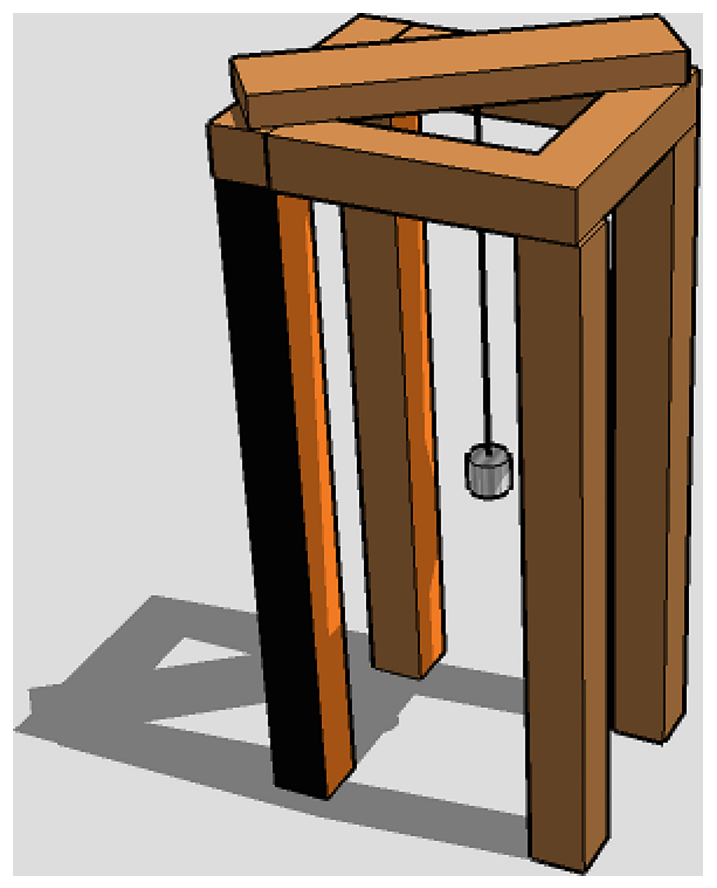

Figura 2: Desenho ilustrativo da estrutura da montagem experimental. A altura da estrutura não é um fator importante e deve ser ajustada de modo a permitir maior comodidade durante a tomada das medidas. 
fio quando esticado verticalmente. Assim quando começa o impacto, a velocidade do bloco é $\sqrt{2 g H}$. A medida experimental da velocidade que causa o rompimento é feita aumentando ou diminuindo a altura da qual a massa $M$ cai até determinar o ponto de transição. Após cada medida, é necessário substituir o fio porque a deformação permanente que ele sofre após o impacto, independentemente dele se romper ou não, o inutiliza.

Os valores apresentados na Tabela 1 para a altura $H$ resultam da média aritmética de três medidas experimentais do ponto de transição de ruptura. Os erros relativos nas medidas das massas e das alturas foram pequenos. Entretanto, os erros estatísticos foram bem maiores e por isso fizemos a estimativa do erro baseado somente no ajuste final dos dados.

\section{Análise teórica}

A análise dimensional juntamente com os dados experimentais permitem expressar a relação entre uma grandeza dependente em função das grandezas independentes na forma mais simples possível. Entretanto, é desejável propor um modelo teórico que permita reproduzir os resultados obtidos experimentalmente. Assim propomos um modelo para o problema do impacto. Suponhamos que a tensão do fio após o impacto seja dada por uma função $T(x)$ até a ruptura, onde $x$ representa a deformação do fio. Antes do impacto a tensão do fio é nula e após o impacto a massa $M$ deforma o fio enquanto a tensão se torna diferente de zero. Se a tensão do fio aumentar suficientemente à medida que a massa $M$ se desloca, então a velocidade do bloco pode se anular antes que o bloco alcance a deformação de ruptura e, nesse caso, ele para e o fio não se rompe. Em caso contrário, o bloco se desloca além do deslocamento de ruptura e o fio se rompe. A massa

Tabela 1: Medidas da massa $M$ que causa a ruptura de um fio de cobre de diâmetro $(0,30 \pm 0,03) \mathrm{mm}$ e $80,0 \pm 0,1$ $\mathrm{cm}$ de comprimento em uma dada altura $H$. Para cada valor de $M$ o valor de $H$ é a média de três alturas medidas.

\begin{tabular}{cc}
\hline$(M \pm 0,1) \mathrm{g}$ & $(H \pm 0,05) \mathrm{cm}$ \\
\hline 1540,0 & 2,85 \\
1220,0 & 3,33 \\
1000,0 & 3,80 \\
670,0 & 13,25 \\
490,0 & 19,70 \\
300,0 & 28,50 \\
210,0 & 44,50 \\
\hline
\end{tabular}

crítica que causa o rompimento do fio é a que leva o bloco à velocidade nula quando o deslocamento é igual à deformação de ruptura. Após o impacto, as forças que atuam no bloco são a força do fio e seu próprio peso. Nesse caso, usando o teorema do trabalho-energia $W=\Delta T$ obtemos a seguinte equação:

$$
-\frac{1}{2} M V^{2}=M g d_{R}-\int_{0}^{d_{R}} T(x) d x .
$$

Para o cálculo do trabalho total realizado pelas forças aplicadas, no segundo membro em (13), das forças aplicadas consideramos o eixo orientado positivamente para baixo, de modo que o trabalho do peso é positivo e o trabalho da tensão é negativo. Explicitando a massa $M$, obtemos

$$
M=\frac{2 \int_{0}^{d_{R}} T(x) d x}{V^{2}+2 g d_{R}} .
$$

Supondo que a tensão de deformação seja proporcional a $x$, ou seja, $T(x)=\frac{T_{R}}{d_{R}} x$, podemos calcular facilmente a integral no numerador da equação (14). Obtemos assim:

$$
M=\frac{T_{R} d_{R}}{V^{2}+2 g d_{R}} .
$$

Nesse caso, a equação (15) tem a forma determinada pela análise dimensional. Lembrando que a velocidade no instante do impacto é dada por $V=\sqrt{2 g H}$, podemos expressar 15) em termos da altura de queda da massa $M$ acima do ponto de impacto, ou seja,

$$
M=\frac{T_{R} d_{R}}{2 g\left(H+d_{R}\right)}
$$

A equação 16 nos será útil na próxima seção.

\section{Análise dos dados}

Para interpretar os dados experimentais obtidos, em primeiro lugar, construímos um gráfico, representado na Figura 3, das medidas apresentadas na Tabela 1. Por comodidade, resolvemos usar a altura da massa $M$ como variável independente.

Reescrevendo (16) na forma

$$
M=\frac{a}{1+b H}
$$

onde

$$
a=\frac{T_{R}}{2 g}=\frac{M_{R}}{2}
$$




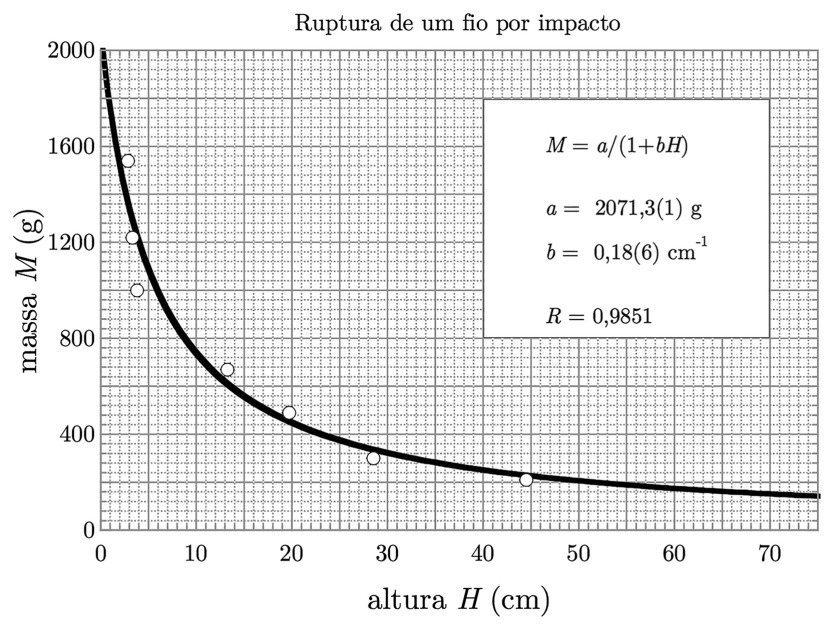

Figura 3: Representação gráfica dos dados experimentais da ruptura de fio por impacto.

e

$$
b=\frac{1}{d_{R}}
$$

onde, por conveniência, definimos $M_{R}=T_{R} / g$ que corresponde a maior massa que o fio pode manter suspensa sem se romper. A curva mostrada no gráfico da Figura (3) corresponde a um ajuste feito com o modelo teórico dado pela equação 17 , o que nos permite determinar os valores dos parâmetros daquela equação.

$$
a=2071,3(1) \mathrm{g} \quad \text { e } \quad b=0,18(6) \mathrm{cm}^{-1} .
$$

Finalmente calculamos, a partir de (18) e (19), a deformação de ruptura $d_{R}=6(2) \mathrm{cm}$ e a massa de ruptura $M_{R}=4142,6(2)$ g. Após o rompimento do fio, medimos os comprimentos dos dois pedaços deformados e calculamos a deformação total do fio. A média obtida para a deformação de ruptura foi de $6,5(1) \mathrm{cm}$. Faremos uma breve discussão sobre essa diferença nas conclusões.

\section{Conclusões}

Os dados experimentais mostrados no gráfico da Figura (3) podem ser bem ajustados a uma curva da forma (17), o que permite obter o valor do deslocamento de ruptura $d_{R}$. Esse fato, contudo, não significa que a linearidade entre a tensão e a deformação seja correta, pois de acordo com a equação (14) observamos que qualquer que seja a lei de força $T=T(x)$ obteremos o mesmo denominador, garantindo assim a forma da curva no ajuste gráfico. Isso significa que, apesar de podermos obter o valor do numerador da equação (14), não podemos extrair informações detalhadas sobre a tensão de ruptura sem mais dados experimentais. Por essa razão e, principalmente, porque se trata de um artigo voltado para o ensino, fizemos a hipótese simplificadora que o trabalho da força de tensão do fio deve-se, principalmente, à região linear. A discrepância observada entre os dois valores da deformação de ruptura obtidos na seção anterior também pode ser atribuída à utilização desse modelo simplificado.

\section{Agradecimentos}

Os autores agradecem aos professores M. Pires do ICT/UFVJM, A.C. Tort e V. Soares do IF/UFRJ pelos comentários e sugestões que ajudaram a elaborar esse trabalho.

\section{Referências}

[1] A.A. Sonin, The Physical Basis of Dimensional Analysis (Department of Mechanical Engineering, Cambridge, 1992), disponível em http://web.mit edu/2.25/www/pdf/DA_unified.pdf, acesso em $9 / 4 / 2015$.

[2] I. Lira, Eur. J. Phys 34, 139 (2013).

[3] L.I. Sedov, Similarity and Dimensional Methods in Mechanics (Academic Press, New York, 1959).

[4] F.L. Carneiro, Análise Dimensional e Teoria da Semelhança e dos Modelos Físicos (Editora UFRJ, Rio de Janeiro, 1993). 